\title{
Exame para Ingresso na Residência Médica de Coloproctologia. A Experiência do Hospital Sírio Libanês (São Paulo, SP)
}

\author{
Resident Selection for Coloproctology - The Sirio Libanes \\ Hospital Experience
}

\author{
RAULCUTAIT ${ }^{1}$, PAULO ALBERTO F. P. CORREA ${ }^{2}$, ENIS DONIZETTI DA SILVA ${ }^{3}$, ROBERTO PADILHA $^{4}$ \\ ${ }^{1}$ Professor Associado do Dpto. de Cirurgia da Faculdade de Medicina da Universidade de São Paulo. Coordenador \\ do Programa de Residência Médica em Coloproctologia do Hospital Sírio Libanês:; ${ }^{2}$ Programa de Residência Médica \\ do Hospital Sírio Libanês; ${ }^{3}$ Presidente do COREME do Hospital Sírio Libanês; ${ }^{4}$ Diretor Executivo do Instituto de \\ Ensino e Pesquisa do Hospital Sírio Libanês - SP - Brasil.
}

CUTAIT R; CORREA PAFP; SILVA ED; PADILHA R. Exame para Ingresso na Residência Médica de Coloproctologia. A Experiência do Hospital Sírio Libanês (São Paulo, SP). Rev bras Coloproct, 2006;26(4): 394-398.

RESUMO: Introdução. Um dos problemas principais no acesso aos programas de residência médica de especialidades é definir não apenas quais são os melhores candidatos, mas quais aqueles que, uma vez terminado o treinamento, poderão aproveitar o investimento intelectual e financeiro que lhes foi facultado. $O$ exame de acesso à residência médica de Coloproctologia do Hospital Sírio Libanês, credenciada pela SBCP e pelo MEC, obedeceu às normas definidas pelo MEC. Nossa preocupação foi de criar um mecanismo de seleção que contemplasse mais que o conhecimento do candidato, dandonos oportunidade de melhor avaliar adestramento cirúrgico, capacidade de resolução de problemas, características de personalidade, interesse na especialidade e planos futuros. Assim, foram desenvolvidas provas que pudessem contemplar mais do que conhecimentos. Exame de seleção. Constou de: 1) prova escrita (5 pontos), abrangendo temas gerais de medicina; 2) prova prática (4 pontos), com quatro partes: a) caso clínico; b) discussão de cuidados pré, intra e pós-operatório; c) cirurgia em animais; d) simulação de atendimento de paciente crítico em boneco - aplicada pelos membros do programa de Coloproctologia; 4) entrevista (1 ponto) - realizada pelos membros do programa. Discussão. O modelo de seleção de residentes oficializado pela Comissão Nacional de Residência Médica é calcado quase que exclusivamente nos conhecimentos dos candidatos, uma vez que o peso da entrevista, ao contrário de outros países, pouco permite mudar a classificação do candidato. Dessa forma, há pouca margem para avaliações subjetivas e cognitivas, dentre as quais se destacam a personalidade do indivíduo, sua capacidade resolutiva, sua competência em atuar em equipe. Para sanar essa limitação, foi conduzida prova prática abrangente e interativa, de modo que a entrevista foi, de certa forma, uma continuação da prova prática. A prova prática, nos moldes que foi elaborada, permitiu ampliar a compreensão sobre as características dos candidatos e proporcionou maior flexibilidade para escolha, o que certamente não ocorreria com o único ponto destinado à entrevista. As opiniões dos diversos examinadores que atuaram nas provas práticas e nas entrevistas foram razoavelmente homogêneas para cada candidato, refletindo a adequação da metodologia utilizada. Conclusão. A prova prática, nos moldes elaborados, embora mais trabalhosa, permitiu definir aspectos diferenciais entre os candidatos, facilitou a condução das entrevistas e permitiu realizar uma melhor seleção dos candidatos. Propostas. A partir dessa experiência, os autores propõem à SBCP: 1) que sejam definidos critérios que permitam padronizar, dentro do possível, os exames de acesso às residências por ela aprovados, através de exame abrangente como o aplicado; 2) que se crie um sistema de avaliação dos residentes ao término de seus programas, acoplado à avaliação dos próprios programas.

Descritores: Residência médica; Coloproctologia; Seleção.

Trabalho realizado no Hospital Sírio Libanês, São Paulo, SP.

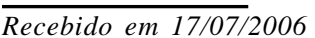

Aceito para publicação em 11/08/2006 


\section{INTRODUÇÃO}

Processos de seleção de recursos humanos são sempre difíceis, uma vez que há a necessidade de, em curto contato, definir qualificações técnicas e pessoais de cada candidato, compará-las às de outros pretendentes e, mais ainda, definir se o candidato preenche os anseios de quem é responsável pela posição desejada. No caso da residência médica, quem seleciona deve não apenas definir os melhores candidatos, mas também identificar aqueles que, uma vez terminado o treinamento, poderão melhor aproveitar o investimento intelectual e financeiro que lhes foi facultado. $\mathrm{O}$ impacto da seleção se faz sentir em diferentes níveis: sobre o próprio candidato, pois se estará abrindo ou não uma porta para sua vida profissional; com o programa de residência e sua instituição, que dependem do bom desempenho do residente; com os pacientes, que não podem correr riscos em função de incompetência ou desinteresse do residente.

De um modo geral, os critérios de seleção para a residência médica são definidos por órgãos ligados ao governo, pelas sociedades médicas ou, então, pelos próprios hospitais, existindo, não raro, a participação dos três níveis mencionados. Na tabela 1 estão listados os principais critérios empregados em diversas modalidades de avaliação dos candidatos à residência.

Em busca de uma maior uniformidade no processo de seleção, empregam-se nos Estados Unidos scores definidos pela USMLE, utilizados também como ferramentas com valor preditivo em relação ao sucesso na carreira ${ }^{1,2}$. Entretanto, preserva-se a diversidade de perfis institucionais, o que gera flexibilização na hora da escolha ${ }^{3,4,5}$. No Brasil, a seleção de residentes era tradicionalmente definida pelos hospitais que, a seu discernimento, utilizavam diferentes pesos para curriculum vitae, prova escrita, prova prática e entrevista. Nos anos 90, apareceram críticas à plena liberdade dos hospitais universitários para elaboração dos exames de residência, uma vez que alguns davam valor ponderal excessivo ao curso médico realizado na faculdade própria, restringindo assim a oportunidade de aprovação para egressos de outros cursos médicos. Isso fez com que a Comissão Nacional de Residência Médica (CNRM), ligada ao MEC, definisse os parâmetros para os exames de seleção a serem aplicados em todo o país, mas de uma forma que gerou uma outra restrição, ou seja, a de supervalorizar o conhecimento, através de uma prova escrita com peso de 9/10, sobrando 1/10 para a entrevista. Em outras palavras, a avaliação do candidato como indivíduo e

Tabela 1 - Critérios para avaliação de candidatos a exames de residência médica.

Competência e conhecimentos

Características pessoais

Avaliação de docentes

Mercado desempenho no internato

notas na faculdade

notas em provas de seleção

experiência na área de interesse

experiência com pesquisa

ética

moral

personalidade

entusiasmo na atividade

capacidade de relacionamento

capacidade de comunicação

capacidade de aprendizado

capacidade resolutiva

sexo

raça

estado civil

aparência

cartas de recomendação

possibilidade de inserção 
sua formação dificilmente conseguiam ter algum impacto no processo de escolha. Há dois anos, foi facultado o exame prático, com peso 4/10, que, de acordo com sua configuração, permite avaliar os candidatos de maneira mais ampla. De qualquer maneira, a avaliação de critérios objetivos, como o curriculum vitae, e subjetivos, dentre eles as características pessoais e os projetos de vida, continua restringida a $1 / 10$ pontos, de modo a ter pouco impacto no critério seletivo.

Preocupados em escolher os residentes da forma mais transparente e adequada possível, desenvolvemos um processo de admissão de residentes para o Programa de Residência Médica em Coloproctologia do Hospital Sírio Libanês observando as orientações da CNRM, mas adaptando-as à nossa visão de processo seletivo.

\section{Residência Médica em Coloproctologia do Hospital Sírio Libanês}

O programa de residência médica em Coloproctologia originou-se a partir de estágios oferecidos informalmente para colegas que procuravam um dos autores (RC) para acompanhar suas atividades clínicas privadas. Em 1997, através de convênio entre o Hospital Sírio Libanês e o Hospital Brigadeiro, da rede pública estadual, um dos autores (RC) passou a coordenar as atividades de Cirurgia Geral e Coloproctologia naquele hospital, o que abriu espaço para ampliar os estágios e dar-lhes uma configuração de residência. Em 2001, juntamente com outros profissionais, foi proposta a criação do Programa de Residência Médica em Coloproctologia (PRM-CP) do Hospital Sírio Libanês, que foi aprovado e oficializado pela Sociedade Brasileira de Coloproctologia em 2001 e pelo MEC em 2002, com duas vagas anuais, com atuação em ambas as instituições.

Um dos pontos cruciais do programa foi definir critérios de seleção transparentes e que atendessem, simultaneamente, às características do grupo envolvido e da instituição. $O$ processo de seleção dos residentes do PRM-CP evoluiu com os anos e, em 2006, testamos um novo modelo, relatado a seguir, que obedeceu à pontuação estabelecida pelo MEC: prova teórica (5 pontos), prova prática (4 pontos) e entrevista (1 ponto). O exame seletivo foi moldado de modo a avaliar e pontuar as seguintes características de cada candidato: 1) o conhecimento, que dá as bases para o aprendizado e para as atividades inerentes de um residente; 2) a capacidade de aprendizado, fundamental para o crescimento técnico e científico; 3) a real motivação para participar do programa; 4) as características de personalidade: caráter, equilíbrio emocional, disciplina. Alguns outros aspectos, tais como vida afetiva e familiar, embora não prioritários e até mesmo delicados ou inoportunos, foram também abordados.

O exame consistiu de três etapas:

Prova teórica. Abrangendo temas gerais da medicina, essa prova foi aplicada simultaneamente aos candidatos dos programas de especialidade do Hospital das Clínicas da Faculdade de Medicina da Universidade de São Paulo e do Hospital Sírio Libanês.

Prova prática. Para o PRM-CP do Sírio Libanês optou-se por prova prática separada da do Hospital das Clínicas e aplicada por membros do grupo e outros médicos do Hospital Sírio Libanês. O intuito era aumentar o contato pessoal entre os examinadores e os candidatos e, assim, conseguir avaliar de forma mais adequada não apenas a competência, mas também algumas características da personalidade e do perfil dos candidatos. A prova prática consistiu de quatro estações, cada uma delas com objetivos definidos:

a) encaminhamento de caso clínico - onde os candidatos exercitaram seu raciocínio clínico, tomando decisões baseadas em dados de anamnese, exame físico e exames laboratoriais e de imagens;

b) discussão de caso clínico - focada na avaliação de conhecimentos básicos relacionados com os cuidados pré, intra e pós-operatórios;

c) cirurgia em animais - objetivou avaliar a capacitação técnica dos candidatos através de procedimentos realizados em porcos, dentre eles: preparação do campo operatório e da mesa de instrumentação; abertura de cavidade; ressecção de segmento de intestino delgado; anastomose intestinal; síntese da parede abdominal;

d) simulação de atendimento de paciente crítico - realizada em estação de simulação em boneco, com o intuito de avaliar conhecimentos e capacidade resolutiva frente a situações de risco de vida.

Entrevista. O tempo de cada entrevista variou de 20 a 30 minutos, tendo se procurado ampliar o conhecimento e entendimento das características pessoais dos entrevistados, seu interesse em participar de nosso programa de residência, motivação para a Coloproctologia, visão quanto a sua futura inserção na medicina e na especialidade. 
Rev bras Coloproct

Outubro/Dezembro, 2006
Exame para Ingresso na Residência Médica de Coloproctologia.

A Experiência do Hospital Sírio Libanês (São Paulo, SP)

Raul Cutait e Cols.
Vol. 26

\section{DISCUSSÃO}

O fortalecimento da Coloproctologia como especialidade passa obrigatoriamente pela competência daqueles que a praticam. Assim, é importante que os programas de residência médica consigam formar bons especialistas, sendo o primeiro passo a correta escolha dos melhores candidatos. Estes podem ser definidos como aqueles que, uma vez terminado o treinamento, irão melhor aproveitar o investimento intelectual e financeiro que lhes foi facultado e exercer a especialidade com competência. Para uma especialidade com apenas 40 programas credenciados no país pela Sociedade Brasileira de Coloproctologia ${ }^{6}$, é fundamental que o processo seletivo seja o mais adequado possível. No modelo americano, existem scores que procuram quantificar todos os indicadores pertinentes. No Brasil, o modelo oficializado pela Comissão Nacional de Residência Médica baseia-se nos conhecimentos dos candidatos, com avaliação teórica quase que invariavelmente realizada por testes de múltipla escolha, o que minimiza a experiência prática do candidato e, no caso da cirurgia, o adestramento cirúrgico, que acabam tendo impacto pouco expressivo na avaliação. Isso gerou uma verdadeira indústria de cursos de preparação para exames de residência médica, que pretendem oferecer condições mais competitivas a candidatos que buscam aumentar seus conhecimentos teóricos, independente de sua desenvoltura na parte clínica.

A recente possibilidade de se realizar também uma prova prática melhorou consideravelmente as condições para quem dedicou seu tempo de internato e residência ao aprendizado prático. Entretanto, as provas práticas que são realizadas em nosso país ainda avaliam, na sua essência, os conhecimentos dos candidatos. Dessa forma, há pouca margem para avaliações subjetivas, dentre as quais se destacam as características de personalidade do indivíduo, sua destreza manual, sua competência em atuar em equipe e a avaliação de seus mentores e professores.

Em 2006, as provas de seleção para residentes em Coloproctologia do Hospital Sírio Libanês foram conduzidas de modo a dar a oportunidade aos candidatos de demonstrarem seus conhecimentos e, aos examinadores, de conhecer o adestramento técnico e as suas características pessoais de cada candidato. Assim, a prova prática foi de fundamental importância para esse processo, por ter sido ampla e permitir con- tato intenso com os candidatos e, de certa forma, a entrevista transformou-se em continuação da prova prática. Portanto, em resumo, o conjunto das avaliações permitiu não só aferir conhecimentos dos candidatos, mas também entendê-los como personalidade e futuros especialistas.

No discernimento de todos que participaram do processo seletivo, foi a prova prática que serviu para sentir "o calor da batalha" e permitir que se conhecesse melhor as características de cada um dos candidatos, de modo a pontuá-los de maneira mais integral, e não apenas em cima dos conhecimentos técnico e científico. Em outras palavras, a prova prática, nos moldes que foi elaborada, proporcionou maior flexibilidade para escolha, o que certamente não ocorreria com o único ponto destinado à entrevista. É interessante notar que as opiniões dos diversos examinadores foram razoavelmente homogêneas para cada candidato, refletindo a adequação da metodologia utilizada.

\section{CONCLUSÕES}

1. O exame de acesso ao PRM-CP do Hospital Sirio Libanês no modelo praticado em 2006 permitiu avaliar os candidatos de maneira mais aprofundada, permitindo uma seleção que pareceu mais adequada a todos os examinadores.

2. A prova prática permitiu definir aspectos diferenciais entre os candidatos, permitindo inclusive uma melhor avaliação pela entrevista.

\section{PROPOSTAS}

1. Continuar a examinar os candidatos ao PRM-CP do Hospital Sírio Libanês dentro do modelo iniciado neste ano e rever a qualidade de nossa avaliação ao término do período de treinamento dos residentes.

2. Levar à Comissão de Ensino da Sociedade Brasileira de Coloproctologia essa experiência e sugerir que sejam criados critérios que permitam padronizar, dentro do possível, os exames de acesso às residências em Coloproctologia aprovadas pela Sociedade.

2. Caso isso aconteça, sugere-se que seja criado um sistema de avaliação dos residentes ao término de seus programas, acoplado à avaliação dos próprios programas. 


\begin{abstract}
Introduction. The major question regarding the access to residency programs is how to properly select the candidates in order to choose those who will better return to society the intellectual and financing investment given to them. The Program in Coloproctology of Hospital Sirio Libanês decided to make an exam to favor not only knowledge, but surgical skills, capability of solving problems, capability of being team players, and personality as well. The Ministery of Education model was observed. Selection of candidates. 1) written exam (5 credits), with tests evaluating knowledge on medicine in general; 2) practical tests (4 credits), applied by members of the Program: a) case discussion; b) case discussion focusing pre, intra e post operative care; c) surgery in animals; d) critical care in simulating model; 4) interview (1 credit) - by members of the Program. Discussion. The National Commission for Medical Residence favors knowledge of the candidates, and only one credit is given to personal interview, which differs from other country models. Our Residency Program in Coloproctology decided to carry on a practical exam that allowed interaction among candidates and examiners, as well as the possibility to evaluate surgical skills, capability of problem solving, team player behavior, personality. Therefore, the practical exam helped to better evaluate candidates during the interviews. Conclusion. The practical exam was complex to prepare. However, it was very useful to promote a better evaluation of the candidates. Proposal. Based on this experience, the authors suggest to SBCP: 1) to define selection criteria to be applied to all Brazilian approved programs; 2) to create a resident evaluation system to be applied to all programs.
\end{abstract}

Key words: Residency program; Coloproctology; Selection.

\section{REFERÊNCIAS}

1. Davis JL, Platt LD, Sandhu M, Shapiro F. Evaluating factors in the selection of residents. Acad Med 1995;70:176-177

2. Carmichael KD, Westmoreland JB, Thomas JA, Patterson RM. Relation of residency selection factors to subsequent orthopaedic in-training examination performance. Southern Med J 2005;98:528-532

3. Self DJ, Baldwin Jr D. Should moral reasoning serve as a criterion for student and resident selection? Clin Orthop Rel Res 2000;378:115-123

4. Gilbart MK, Cusimano MD, Regehr G. Evaluating surgical resident procedures. Am J Surg 2001;181:221-225
5. Bajaj G, Carmichael KD. What attributes are necessary to be selected fot na orthopedic surgery residency position: perception of faculty and residents. Southern Med J 2004;97:1179-1185

6. www.sbcp.org.br/serviços credenciados novembro 2006.

Endereço para correspondência:

RAUL CUTAIT

Rua Adma Jafet, 50 / $6^{\circ}$ and.

01308-050 - São Paulo - SP

FAX: 55-11-3255-9397

E-mail: ycutait@hsl.org.br 